This is a post-peer-review, pre-copyedit version of a paper published in He H, Wagner AR, Castro-Gonzalez A, Salichs MA, Ge SS, Barakova EI \& Cabibihan J (eds.) Social Robotics. ICSR 2019. Lecture Notes in Computer Science, 11876. ICSR 2019: International Conference on Social Robotics, Madrid, Spain, 26.11.2019-29.11.2019. Cham, Switzerland: Springer International Publishing, pp. 34-43. The final authenticated version is available online at:https://doi.org/10.1007/978-3-030-35888-4_4

\title{
Spatiotemporal Coordination Supports a Sense of Commitment in Human-Robot Interaction
}

\author{
Alessia Vignolo ${ }^{1,2}$, Alessandra Sciutti ${ }^{3}$, Francesco Rea $^{2}$, and John Michael ${ }^{1}$ \\ 1 Department of Philosophy, Social Sciences Building, University of Warwick, \\ Coventry, CV4 7AL, United Kingdom \\ 2 Robotics Brain and Cognitive Sciences Unit, Istituto Italiano di Tecnologia, Via \\ Enrico Melen 83, 16152 Genoa, Italy \\ ${ }^{3}$ CONTACT Unit, Istituto Italiano di Tecnologia, Via Enrico Melen 83, 16152 \\ Genoa, Italy
}

\begin{abstract}
In the current study, we presented participants with videos in which a humanoid robot (iCub) and a human agent were tidying up by moving toys from a table into a container. In the High Coordination condition, the two agents worked together in a coordinated manner, with the human picking up the toys and passing them to the robot. In the Low Coordination condition, they worked in parallel without coordinating. Participants were asked to imagine themselves in the position of the human agent and to respond to a battery of questions to probe the extent to which they felt committed to the joint action. While we did not observe a main effect of our coordination manipulation, the results do reveal that participants who perceived a higher degree of coordination also indicated a greater sense of commitment to the joint action. Moreover, the results show that participants' sensitivity to the coordination manipulation was contingent on their prior attitudes towards the robot: participants in the High Coordination condition reported a greater sense of commitment than participants in the Low Coordination condition, except among those participants who were a priori least inclined to experience a close sense of relationship with the robot.
\end{abstract}

Keywords: Cognitive human-robot interaction - Sense of commitment . iCub.

\section{Introduction}

As robots become increasingly prevalent throughout everyday life and in domains ranging from health care to education and manufacturing $[2,8,4,15,6]$, researchers are devoting ever more attention to developing new ways of optimizing human-robot-interaction. In the industrial context, for example, there has already been a shift from the use of robots as fully pre-programmed devices performing single predefined tasks towards the adoption of co-bots, able to adapt to new tasks and new human partners. One challenge in this regard is to boost human interactants' willingness to invest time and effort when interacting with a robot partner and in persisting in an interaction as the robot adapts to a new 
context and a new human partner. While there is a risk of human interactants becoming frustrated or impatient when a robot is slow to adapt, the potential benefits of adaptation are high insofar as they can maximize a robot's ability to contribute to new tasks with new partners.

To address this challenge, Powell and Michael [10] (cf. also [11]) have recently proposed that a potentially effective and low-cost strategy could be to develop design features that serve to maintain a human's sense of commitment to an interaction with a robot. By boosting a human agent's sense of commitment, it may be possible to increase her or his willingness to remain patient and persistent when the robot makes errors or is slow to perform a task. To achieve this, they recommend the implementation of features that have been shown to promote a sense of commitment in human-human interaction.

For example, recent research on human-human interaction provides evidence that spatiotemporal coordination may boost a sense of commitment [12] (cf. also [13]), leading people to persist longer and to invest greater effort in joint actions. This is because, when two agents coordinate their contributions to a joint action, they form and implement interdependent, i.e. mutually contingent, action plans. The agents thus form interdependent action plans. Each agent must therefore have - and rely upon - expectations about what the other agent is going to do. This may generate social pressure on the other agent to perform the expected actions. It is not yet known, however, to what extent spatiotemporal coordination may have similar effects in the context of human-robot interaction.

\subsection{Aim of the Study}

In the current study, we extend the research on spatiotemporal coordination and commitment to the context of human-robot interaction. To this end, we adapted a paradigm used in research on human-human joint action [8]. Specifically, we designed and implemented an online study in which we presented participants with videos in which a humanoid robot (iCub [9]) and a human agent were tidying up by moving toys from a table into a container. In the High Coordination condition, the two agents formed a chain, with the human picking up the toys and passing them to the robot. In the Low Coordination condition, they worked in parallel without forming a chain. Participants were asked to imagine themselves in the position of the human agent and to respond to a battery of questions to probe the extent to which they felt committed to the joint action. We predicted that they would indicate a higher degree of commitment in the High Coordination condition than in the Low Coordination condition.

\section{Methods}

We used SurveyMonkey to administer a web-based observational paradigm. Participants were recruited via prolific academic. Since each participant gave only one judgment per condition, and since online experiments produce greater variability than lab-based experiments, we aimed for a large sample size: 100 per 
condition in a between-subjects design. Anticipating that about $25 \%$ of our participants may need to be excluded, we solicited 250 participants and also included an additional nine participants who completed the survey before prolific had registered that the target number had been reached. Each of the 259 participants received a payment of 1 Pound. We excluded 19 participants who either did not complete the experiment or did not correctly answer the question "What items were John and the robot clearing away?". The final dataset was thus composed by 240 participants (129 males, 107 females, 1 other and 2 preferred not to say) between the ages of 16 and $74(M=28.95$ years, $S D=10.52$ years). The experiment was conducted in accordance with the Declaration of Helsinki, and was approved by the Humanities \& Social Sciences Research Ethics Sub-committee (HSSREC) at the University.

\section{$2.1 \quad$ Stimuli}

iCub's movements of all the body parts (arms, torso, neck and eyes) were controlled entirely with the Constant Time Position Service (CTP Service) that takes as input the desired position in joint space and the timing, and conforms to biological motion (minimum jerk model). In both conditions, the robot and the human partner do not perform mutual gaze during the interaction. In both conditions, the robot's gaze anticipates the right hand movement (e.g. in the Low Coordination condition, the robot's gaze anticipates the reaching and the grasping of the object), to conform to biologically-inspired behaviour.

\subsection{Procedure}

After providing their informed consent and answering basic demographic questions, participants are asked to respond to a preliminary question ("Have you ever interacted with a robot?"). Then, participants are shown a brief video (the introductory video) in which the humanoid robot iCub is tidying up by moving toys from a table into a container. In the video, a human agent who is passing by stops and begins to help the robot.

Participants are then asked to imagine being in the position of the human agent, to assess how close they feel to the robot, and to report this using the 7-point Inclusion of Other in the Self (IOS) Scale [19]. (before seeing the video of the joint action and after). Next, a brief text explaining the scenario is shown:

The robot in the picture has the task of cleaning up a bunch of small items from the morning activity. As you will see in a very brief video, John is walking by to get his laptop when he notices the robot cleaning up. Since he is going to need to use this table later on, he stops to help for a bit to ensure that the cleaning up is going well.

Participants then watch a video composed of the introductory video and the video of the joint action (repeated twice) in one of the two conditions: in the Low Coordination condition, the human agent and the robot act independently of each other, with each of them grasping toys individually and putting them into the container (Figure 1, left). In the High Coordination condition, they form a 
chain in which the human grasps toys and passes them to the robot, whereupon the robot drops them into the container (Figure 1, right). In the High Coordination condition, the video lasts about 40 seconds; in the Low Coordination condition, the video lasts 37 seconds. In both conditions, the overall number of toys placed in the box is 4 (in the Low Coordination condition each agent puts two toys into the container; in the High Coordination condition all four toys are passed from the human to the robot).

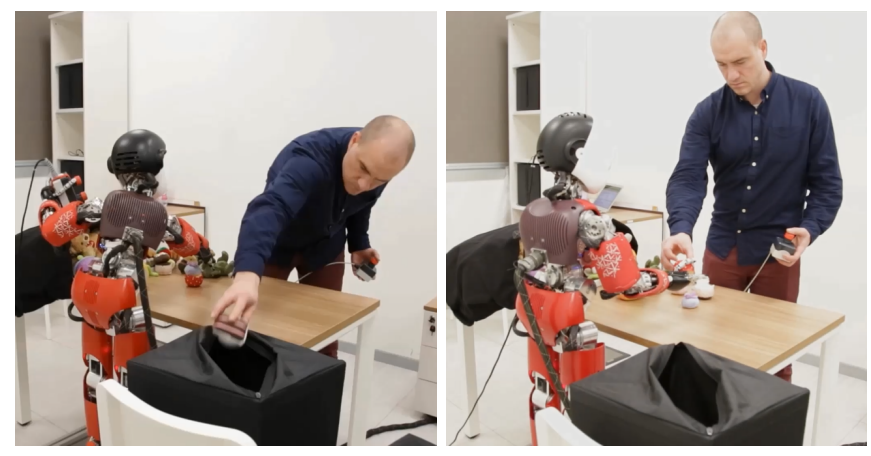

Fig. 1. Screenshots of the video stimuli: Low Coordination (left) and High Coordination condition (right).

After the video, participants are asked the following questions:

- The "persistence question": How long would you keep helping if you were in John's place? (1, Not at all - 5, Until all objects are cleared away)

- The "resistance to distraction question": If you were in John's place and your phone were to start ringing...how likely is that you would take the call? (1, Highly likely - 4, Highly unlikely)

- The "patience question": Sometimes the robot has to stop to recalibrate its sensors. This requires that the entire activity be stopped and can take anywhere from a few seconds to 10 minutes. If you were in John's place and the robot had to pause to recalibrate, how long do you think you would wait for him before giving up and leaving? (1, Not at all - 6, As long as it takes)

- The "coordination question": To what extent did John and the robot seem to be coordinating with each other? ( 1 , Not at all - 5, Completely)

- The "human coordination question": To what extent did John seem to be coordinating with the robot? (1, Not at all - 5 , Completely)

- The "robot coordination question": To what extent did the robot seem to be coordinating with John? (1, Not at all - 5, Completely)

- The "attention check question": What items were John and the robot clearing away?

The persistence question, the resistance to distraction question and the patience question all operationalize commitment. They enable us to assess whether 
participants that were exposed to the High Coordination condition would continue longer, would be less likely to be distracted by an external stimulus, and would wait longer if the robot had to stop.

The three coordination questions enable us validate the stimuli and to demonstrate that participants in the High Coordination condition indeed perceived a higher degree of coordination. Moreover, they also enable us to probe whether participants perceived the coordination to be driven more by the robot or by the human.

The attention check question about the items cleared away enables us to identify and exclude participants who had not watched the video carefully. We did not insert an attention check question about the coordination as we did not want to force participants to observe a coordination between agents if they did not notice it.

\section{Results}

For the persistence question, we observed a quantitative difference such that participants in the High Coordination condition indicated that they would help for longer $(M=3.36, S D=1.53)$ than participants in the Low Coordination condition $(M=3.07, S D=1.43)$, but an independent $t$-test revealed no significant difference, $t(238)=1.477, p=0.141$. For the resistance to distraction question, we did not observe a significant difference between participants in the High Coordination condition $(M=1.47, S D=0.64)$ and participants in the Low Coordination condition $(\mathrm{M}=1.45, S D=0.71)$, independent $t$-test $t(238)$ $=0.176, p=0.860$. For the patience question, participants in the High Coordination condition indicated that they would help longer $(M=3.31, S D=1.49)$ than participants in the Low Coordination condition $(M=3.01, S D=1.35)$, and an independent $t$-test revealed a marginal effect, $t(238)=1.661, p=0.098$.

As a manipulation check, we analysed the data from the coordination question and the robot coordination question. Regarding the coordination question, participants who observed the High Coordination condition video perceived a higher degree of coordination between the human and the robot $(M=4.09, S D$ $=0.88)$ than participants who observed the Low Coordination condition video $(M=3.29, S D=1.12)$, and an independent $t$-test revealed a significant effect, $t(238)=6.207, p=2.382 \cdot 10^{-9}$.

Regarding the robot coordination question, participants who observed the High Coordination condition video perceived a higher degree of coordination on the part of the robot $(M=3.96, S D=1.05)$ than participants who observed the Low Coordination condition video $(M=2.68, S D=1.27)$, and an independent $t$-test revealed a significant effect, $t(238)=8.488, p=2.284 \cdot 10^{-15}$.

We then conducted a series of one-way ANCOVAs with the perceived coordination (coordination question) as covariate (Figure 2). For the persistence question, it revealed a significant effect of the covariate on perceived commitment, $\mathrm{F}(1,237)=13.62, p=0.0003$, but no significant effect of the condition, $\mathrm{F}(1,237)=0.02, p=0.884$, or of the interaction, $\mathrm{F}(1,237)=2.72, p=0.100$. 
For the resistance to distraction question, it revealed no significant effect of the covariate, $\mathrm{F}(1,237)=1.11, p=0.293$, of the condition, $\mathrm{F}(1,237)=0.06, p=$ 0.815 or of the interaction, $\mathrm{F}(1,237)=1.78, p=0.183$. For the patience question, it revealed a significant effect of the covariate on the perceived commitment, $\mathrm{F}(1$, $237)=15.27, p=0.0001$, but no significant effect of the condition, $\mathrm{F}(1,237)=$ $0.07, p=0.796$, or of the interaction, $\mathrm{F}(1,237)=0.34, p=0.562$.

We also conducted a series of one-way ANCOVAs with the perceived coordina-
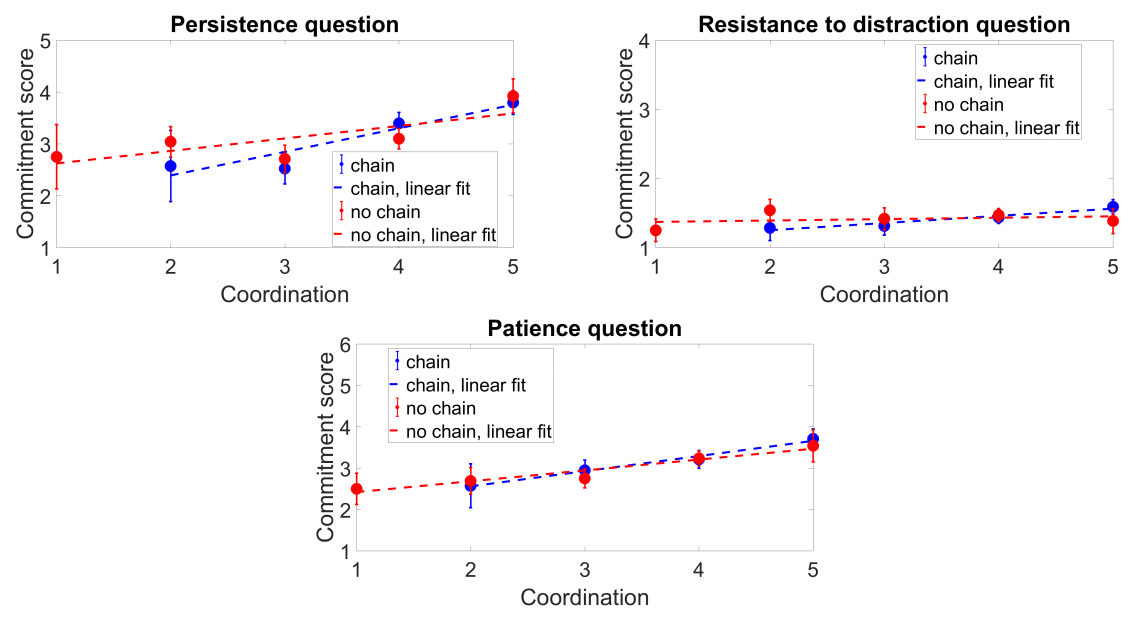

Fig. 2. Effect of condition (High Coordination vs. Low Coordination), with perceived coordination (i.e. response to the coordination question) as covariate, on perceived commitment (i.e. responses to the persistence question, the resistance to distraction question, and the patience question).

tion of the robot (i.e. responses to the robot coordination question) as covariate. For the persistence question, it revealed a significant effect of the covariate on perceived commitment, $\mathrm{F}(1,237)=6.21, p=0.013$, but no significant effect of the condition, $\mathrm{F}(1,237)=0.11, p=0.747$, or of the interaction, $\mathrm{F}(1,237)=$ $0.65, p=0.423$. For the resistance to distraction question, it revealed no significant effect of the covariate, $\mathrm{F}(1,237)=2.35, p=0.127$, of the condition, $\mathrm{F}(1,237)=0.41, p=0.522$, or of the interaction, $\mathrm{F}(1,237)=0.55, p=0.457$. For the patience question, it revealed a significant effect of the covariate on the perceived commitment, $\mathrm{F}(1,237)=24.27, p=1.58 \cdot 10^{-6}$, but no significant effect of the condition, $\mathrm{F}(1,237)=0.54, p=0.462$, or of the interaction, $\mathrm{F}(1$, $237)=0.004, p=0.947$.

Next, we probed whether participants' responses to the three commitment questions varied according to the closeness which they reported to the robot prior to the experiment. To do so, we conducted a series of one-way ANCOVAs with closeness as covariate. For the persistence question, it revealed a significant 
effect of the interaction between the condition and the covariate on perceived commitment, $\mathrm{F}(1,237)=6.43, p=0.012$, but no significant effect of the covariate, $\mathrm{F}(1,237)=1.30, p=0.255$, or of the condition, $\mathrm{F}(1,237)=2.26, p=$ 0.134 . For the resistance to distraction question, it revealed a marginal effect of the covariate, $\mathrm{F}(1,237)=3.68, p=0.056$, no significant effect of the condition, $\mathrm{F}(1,237)=0.04, p=0.847$, or of the interaction, $\mathrm{F}(1,237)=0.03, p=0.857$. For the patience question, it revealed a marginal effect of the covariate, $\mathrm{F}(1$, $237)=2.75, p=0.099$, of the condition, $\mathrm{F}(1,237)=2.86, p=0.092$, and of the interaction, $\mathrm{F}(1,237)=3.29, p=0.071$,

As illustrated in Figure 3, participants who indicated that they felt least close with the robot (i.e. responded with a ' 1 ') were least sensitive to our manipulation. In fact, for the persistence question, they exhibit a trend in the direction opposite to our hypothesis: they are more willing to persist in interacting with the robot when there is not a high degree of coordination. The rest of the participants exhibit a pattern of responses that is consistent with our hypothesis.

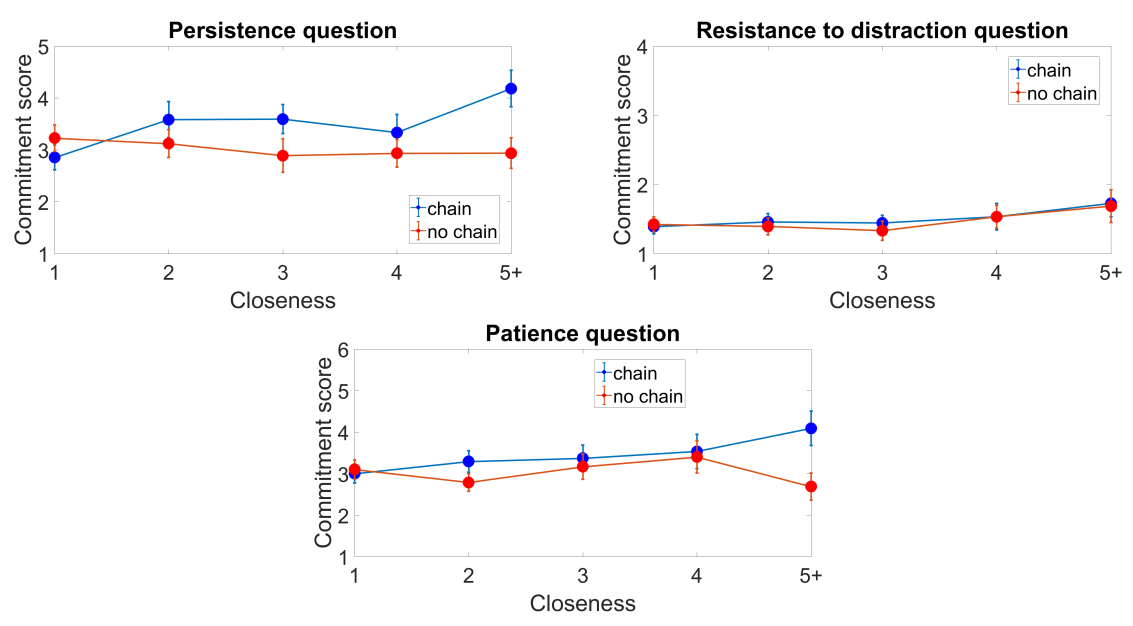

Fig. 3. Effect of the closeness which they reported to the robot prior to the experiment on perceived commitment (i.e. responses to the persistence question, the resistance to distraction question, and the patience question).

A linear regression was calculated to predict perceived commitment (i.e. responses to the persistence question, the resistance to distraction question, and the patience question) based on perceived coordination (i.e. responses to the coordination question). For the persistence question, a significant regression equation was found $(\mathrm{F}(1,238)=13.57, p=0.0003)$, with an $R^{2}$ of 0.054 . Participants' perception of commitment was equal to $2.039+0.318$ (perceived coordination score). Commitment increased 0.318 for each point of perceived coordination. For the resistance to distraction question, no significant regression equation was 
found $(\mathrm{F}(1,238)=1.112, p=0.293)$, with an $R^{2}$ of 0.005 . Participants predicted commitment is equal to $1.3026+0.042$ (perceived coordination score). Commitment increased 0.042 for each point of perceived coordination score. For the patience question, a significant regression equation was found $(\mathrm{F}(1,238)=15.37$, $p=0.0001)$, with an $R^{2}$ of 0.061 . Participants' perception of commitment was equal to $1.961+0.325$ (perceived coordination score). Commitment increased 0.325 for each point of perceived coordination.

A linear regression was calculated to predict perceived commitment (i.e. responses to the persistence question, the resistance to distraction question, and the patience question) based on perceived robot's coordination (i.e. responses to the robot coordination question). For the persistence question, a significant regression equation was found $(\mathrm{F}(1,238)=6.245, p=0.013)$, with an $R^{2}$ of 0.026. Participants predicted commitment is equal to $2.621+0.179$ (perceived coordination score). Commitment increased 0.179 for each point of perceived coordination score. For the resistance to distraction question, no significant regression equation was found $(\mathrm{F}(1,238)=2.358, p=0.126)$, with an $R^{2}$ of 0.010 . Participants predicted commitment is equal to $1.293+0.050$ (perceived coordination score). Commitment increased 0.050 for each point of perceived coordination score. For the patience question, a significant regression equation was found $\left(\mathrm{F}(1,238)=24.41, p=1.465 \cdot 10^{-6}\right)$, with an $R^{2}$ of 0.093 . Participants predicted commitment is equal to $2.072+0.328$ (perceived coordination score). Commitment increased 0.328 for each point of perceived coordination score.

\section{Discussion}

Current research in HRI is more and more devoted to trying to obtain seamless and pleasant interactions and transfer of skills between humans and robots [16, 1]. However, extended interaction also requires patience from human partners in face of errors or simply to slow robot behaviours. Previous work in robotics has investigated how to deal with errors or lack of knowledge, suggesting different strategies for robots to ask the right questions [3] or to be transparent about its errors in order to mitigate negative human reactions - and to reduce the risk of humans abandoning the interaction [5]. In this work we explored a different approach, testing the hypothesis that a high degree of spatiotemporal coordination promotes a sense of commitment in the context of human robot interaction. To this end, participants were instructed to imagine themselves in the role of a human agent interacting with a humanoid robot either with a high degree of spatiotemporal coordination (High Coordination condition) or with a low degree of spatiotemporal coordination (Low Coordination condition), and asked to respond to a battery of questions to probe the extent to which they felt committed to the joint action. We predicted that they would indicate a greater sense of commitment in the High Coordination condition than in the Low Coordination condition. Though we did not observe a main effect of our coordination manipulation, the exploratory analyses did reveal that those participants who perceived a higher degree of coordination - and particularly those participants 
who perceived that the robot was actively coordinating with the human agent also indicated a greater sense of commitment to the joint action. This is consistent with our hypothesis, though it suggests that our stimuli may not have been optimally designed to present contrasting degrees of coordination. The results also show that participants' sensitivity to the coordination manipulation was contingent on their prior attitudes towards the robot: participants in the High Coordination condition reported a greater sense of commitment than participants in the Low Coordination condition, except among those participants who were a priori least inclined to experience a close sense of relationship with the robot.

Our findings contribute to a growing body of research investigating how simple design features may be used to elicit a human agent's sense of commitment to a joint action with a robot, thereby increasing the human's willingness to remain patient and invest effort in the joint action. In particular, Székely et al. (forthcoming; cf. also Vignolo, under review) have shown that noticing a robot's investment of effort in a joint action, beyond improving subjects' performance [17], can boost a human agent's sense of commitment, thereby enhancing their persistence and patience. The current findings extend this research by providing preliminary evidence that a second factor, namely spatiotemporal coordination, may have similar effects. This provides roboticists with a further tool to use in designing robots that can elicit a sense of commitment on the part of human agents. These results also suggest that developing new approaches to enable robots to detect sensorimotor regularities in human partners' behaviours [18] and adapt to them $[14,7]$ could bring in advantages beyond simple efficiency, potentially also leading to higher degrees of commitment toward robotic partners. It would be valuable for further research to explore the effects of spatiotemporal coordination in other contexts and with other tasks. Moreover, an important next step would be to investigate the effects that spatiotemporal coordination with a robot has upon the sense of commitment when people are directly involved in the interaction rather than watching a video and imagining themselves in the interaction.

\section{Acknowledgment}

This research was supported by a Starting Grant from the European Research Council (nr. 679092, SENSE OF COMMITMENT).

\section{References}

1. Argall, B.D., Chernova, S., Veloso, M., Browning, B.: A survey of robot learning from demonstration. Robotics and Autonomous Systems 57(5), 469-483 (2009)

2. Breazeal, C., Brooks, A., Gray, J., Hoffman, G., Kidd, C., Lee, H.: Humanoid robots as cooperative partners for people. Journal of Humanoid Robots p. 34 (2004)

3. Cakmak, M., Thomaz, A.L.: Designing robot learners that ask good questions. In: Proceedings of the Seventh Annual ACM/IEEE International 
Conference on Human-Robot Interaction. pp. 17-24. HRI '12, ACM, New York, NY, USA (2012). https://doi.org/10.1145/2157689.2157693, http://doi.acm.org/10.1145/2157689.2157693

4. Clodic, A., Cao, H., Alili, S., Montreuil, V., R., A., R., C.: Shary: A supervision system adapted to human-robot interaction. Experimental Robotics. Springer Berlin Heidelberg pp. 229-38 (2009)

5. Fischer, K., Weigelin, H., Bodenhagen, L.: Increasing trust in human-robot medical interactions: effects of transparency and adaptability. Paladyn, Journal of Behavioral Robotics 9(1), 95-109 (2018)

6. Grigore, E., Eder, K., Pipe, A., Melhuish, C., Leonards, U.: Joint action understanding improves robot-to-human object handover. IEEE/RSJ International Conference on Intelligent Robots and Systems pp. 4622-9 (2013)

7. Iqbal, T., Rack, S., Riek, L.D.: Movement coordination in humanrobot teams: A dynamical system approach. IEEE Transactions on Robotics (2016)

8. Lenz, C., Nair, S., Rickert, M., Knoll, A., Rosel, W., Gast, J.: Joint-action for humans and industrial robots for assembly tasks. IEEE pp. 130-5 (2008)

9. Metta, G., Natale, L., Nori, F., Sandini, G., Vernon, D., Fadiga, L., Von Hofsten, C., Rosander, K., Lopes, M., Santos-Victor, J., et al.: The icub humanoid robot: An open-systems platform for research in cognitive development. Neural Networks 23(8-9), 1125-1134 (2010)

10. Michael, J., Powell, H.: Feeling committed to a robot: Why, what, when, and how? Philosophical Transactions of the Royal Society B: Biological Sciences p. 374 (1771) (2019)

11. Michael, J., Salice, A.: The sense of commitment in human-robot interaction. International Journal of Social Robotics 9(5), 75563 (2017)

12. Michael, J., Sebanz, N., Knoblich, G.: Observing joint action: Coordination creates commitment. Cognition pp. 106-113 (2016)

13. Michael, J., Sebanz, N., Knoblich, G.: The sense of commitment: A minimal approach. Frontiers in Psychology 6(1968) (2016)

14. Rea, F., Vignolo, A., Sciutti, A., Noceti, N.: Human motion understanding for selecting action timing in collaborative human-robot interaction. Frontiers in Robotics and AI (2019)

15. Sciutti, A., Bisio, A., Nori, F., Metta, G., Fadiga, L., Pozzo, T.: Measuring human-robot interaction through motor resonance. International Journal of Social Robotics 4(3), 223-34 (2012)

16. Sciutti, A., Mara, M., Tagliasco, V., Sandini, G.: Humanizing human-robot interaction: On the importance of mutual understanding. IEEE Technology and Society Magazine 37(1), 22-29 (2018)

17. Vignolo, A., Powell, H., McEllin, L., Rea, F., Sciutti, A., Michael, J.: An adaptive robot teacher boosts a human partner's learning performance in joint action. The 28th IEEE International Conference on Robot \& Human Interactive Communication (RO-MAN 2019), New Delhi, India (2019)

18. Vignolo, A., Noceti, N., Rea, F., Sciutti, A., Odone, F., Sandini, G.: Detecting biological motion for human-robot interaction: A link between perception and action. Frontiers in Robotics and AI 4, 14 (2017)

19. Woosnam, K.M.: The inclusion of other in the self (ios) scale. Ann Tourism Res. 37, 857-60 (2010) 